\title{
Does pain in the masseter and anterior temporal muscles influence maximal bite force?
}

\author{
Marcelo Coelho Goiato ${ }^{\mathrm{a}}$, Paulo Renato Junqueira Zuimª, Amália Moreno ${ }^{\mathrm{b}}$, \\ Daniela Micheline dos Santos ${ }^{\mathrm{a}}$, Emily Vivianne Freitas da Silva ${ }^{\mathrm{a}}$, Fernanda Pereira de Caxias ${ }^{\mathrm{a}}$, \\ Karina Helga Leal Turcio ${ }^{a, *}$ \\ a Department of Dental Materials and Prosthodontics, Aracatuba Dental School, Sao Paulo State University (UNESP), Brazil \\ b Department of Oral Surgery, Pathology and Dental Clinical, School of Dentistry, Federal University of Minas Gerais (UFMG), Brazil
}

\section{A R T I C L E I N F O}

\section{Keywords:}

Temporomandibular joint disorders

Bite force

Occlusal splints

Bruxism

\begin{abstract}
A B S T R A C T
Objective: The aim of this study was to evaluate changes in pain and muscle force, and the relationship between them, in patients with muscle pain and bruxism, prior to and after treatment.

Methods: Thirty women with bruxism and myofascial pain (Ia) were included in this study. Sleep bruxism diagnosis was made based on clinical diagnostic criteria, and awake bruxism diagnosis was made by patient questionnaires and the presence of tooth wear. The diagnosis of myofascial pain was established according to the Research Diagnostic Criteria for Temporomandibular Disorders (RDC-TMD). Dentulous or partially edentulous patients (rehabilitated with conventional fixed prostheses) were included in the study according to the inclusion and exclusion criteria. The pain treatment protocol included occlusal splints, patient education, and physiotherapy for 30 days. Bite force was measured using a dynamometer at the central incisor and the first molar regions on both sides. The exams were performed at baseline, after 7 days, and 30 days after treatment. The Wilcoxon test was used to compare patient pain level response among the periods analyzed in the study. Bite force data were submitted to two-way repeated-measures ANOVA, followed by the Tukey HSD test $(p<0.05)$. A simple regression analysis was performed to verify the relation between pain level and bite force.

Results: Results revealed that there was a statistical difference in pain level over time for both muscles and sides $(p<0.01)$. In the molar region, the bite force exhibited significantly higher values after 30 days of treatment, when compared with the baseline $(p<0.001)$. There was a correlation between pain level and bite force only for the temporal muscle in all periods analyzed $(\mathrm{p}<0.05)$. There was no strong correlation in the response level points to support the association of pain and bite force.

Conclusions: Pain level decreased and bite force increased in the molar region after treatment. No strong correlation or dispersion in the relationship between pain levels and bite force was seen in women with myofascial pain and bruxism.
\end{abstract}

\section{Introduction}

Myogenous pain is frequently reported in patients with temporomandibular disorder (TMD) (de Leeuw \& Klasser, 2013; Svensson, Burgaard, \& Schlosser, 2001). Lobbezoo et al. (2013) defined bruxism as a repetitive activity of the jaw muscles characterized by clenching of the teeth, associated or not associated with bracing of the mandible. This condition has two circadian manifestations, since it can occur during sleep (sleep bruxism) or while awake (awake bruxism).

Bruxism diagnosis can be performed by using polysomnography, physical exams, and questionnaires (Ahlberg et al., 2008; Paesani et al.,
2013). Polysomnography associated with an audio-video system is still the gold standard for this type of evaluation, but it requires time and is expensive (Lavigne et al., 2000). Generally, questionnaires are used for research and clinical exams are used to detect awake and sleep bruxism. Although questionnaires are subjective, their main advantage is that they can be applied to a large population (Lobbezoo et al., 2013).

Different treatment alternatives, such as physiotherapy, medication, therapies against emotional stress, and occlusal splints, have been indicated for patients with painful TMD and bruxism (Hamata, Zuim, \& Garcia, 2009; Gomes, El Hage, Amaral, Politti, \& BiasottoGonzalez, 2014; Wahlund, Nilsson, \& Larsson, 2015). Some authors

\footnotetext{
*Corresponding author at: Department of Dental Materials and Prosthodontics, Aracatuba Dental School, Sao Paulo State University (UNESP), José Bonifácio, 1193, Vila Mendonca, Araçatuba, São Paulo 16015-050, Brazil.

E-mail addresses: karina@foa.unesp.br, luisfernandoc@ymail.com (K.H.L. Turcio).
} 
affirm the short-term effectiveness of the treatments mentioned to recover neuromuscular harmony in the masticatory system (Wahlund et al., 2015), as well as their role in tooth wear protection and a possible decrease in muscle activity during sleep (Klasser, Greene, \& Lavigne, 2010). Gomes et al. (2014) demonstrated that the use of occlusal splints associated with physical therapy did not decrease the electrical activity in masseter or anterior temporal muscles, but did reduce the intensity of symptoms and signs among severe TMD patients with sleep bruxism.

In dentistry, bite force level has been used to analyze the performance of prosthetic rehabilitations, and to provide reference values for studies on masticatory muscles (Fernandes, Glantz, Svensson, \& Bergmark, 2003; Mancuso, Goiato, Gennari Filho, \& Gomes, 2008; Shimada, Baad-Hansen, \& Svensson, 2015). English, Buschang, and Throckmorton (2002) and Abreu et al. (2014) affirmed that a sufficient bite force is an indicator of normal masticatory function. Bite force level is related to a number of variables, such as craniomandibular anatomy, facial dimensions (Gomes et al., 2014), gender (Koç, Doğan, \& Bek, 2011; Palinkas et al., 2010), age (Palinkas et al., 2010), periodontal support of teeth (Okada et al., 2014), temporomandibular disorders, and pain (Pereira-Cenci, Pereira, Cenci, Bonachela, \& Del Bel Cury, 2007). A study comparing patients with TMD and healthy individuals demonstrated a lower bite force in the TMD patients. However, no differences were found in maximal bite force results between the TMD and healthy (control) groups (Pereira-Cenci et al., 2007).

Research showed that the variation in the intensity of bite force could be due to different factors such as gender, age, periodontal aspect, and health of the masticatory system (Okada et al., 2014; ; Pereira et al., 2007). Although there are published studies about bite force in TMD patients, results are still unclear since these studies did not evaluate patients that only complained about muscle pain.

The aim of this study was to evaluate the changes in pain and muscle force, and the relationship between them, in patients with muscle pain and bruxism, prior to and after 30 days of treatment with occlusal splints, patient education, and physiotherapy. The research null hypotheses were that the pain level and bite force do not vary over time, and that there is no relationship between pain level and bite force at the baseline and after treatment.

\section{Materials and methods}

\subsection{Selection of patients and muscle palpation}

The research protocol was approved by the Human Research Ethics Committee (Process CEP/286.401), in accordance with the principles of the Helsinki Declaration. Patients were informed about the study and signed an informed consent form.

Anamnesis and clinical exams of 102 patients requiring TMD treatment were performed at the Aracatuba Dental School between April 2013 and June 2013. The sample size was defined considering the following levels (ANOVA statistical test): a 0.05 level of significance, $80 \%$ power, and a medium effect size. In addition, an intra-individual correlation coefficient of 0.75 was considered (Faul, Erdfelder, Lang, \& Buchner, 2007). The results of the calculation showed that 21 individuals were required to achieve $80 \%$ power for the explanatory variables. Fifty-five patients were diagnosed with muscular TMD and bruxism. Therefore, 30 participants met the inclusion criteria, which was enough to achieve $95 \%$ power and carry out the study.

The inclusion criteria were the presence of myofascial pain, awake and/or sleep bruxism, as well as Angle class I molar relationship and dentate or partially dentate rehabilitated patients with conventional fixed prostheses (absence of no more than 3 teeth in each arch).

Myofascial pain was identified according to the Research Diagnostic Criteria for Temporomandibular Disorders (RDC-TMD) (Dworkin, \& LeResche, 1992). The "probable" awake bruxism was considered based on patient questionnaires and the presence of tooth wear at the clinical exam (Lobbezoo et al., 2013). Sleep bruxism diagnosis was made based on clinical diagnostic criteria (Pintado, Anderson, DeLong, \& Douglas, 1997) proposed by the American Academy of Sleep Medicine (AAMS).

The following exclusion criteria were adopted: a) presence of any systemic pathology; b) use of illegal drugs, antidepressant medications, or sedatives; c) use of removable prostheses; d) patients scheduled for dental procedures that could alter the occlusion during therapy; and e) TMD of articular origin.

Thirty 30 patients were included in the study after meeting the criteria cited above. The palpation of masseter and anterior temporal muscles was assessed according to the TMD Clinical Examination Form, prior to and after 30 days of treatment. For the analyses, the regions of greatest pain intensity in each muscle (anterior temporal and masseter) were considered, and the palpation pain on muscle palpation was classified according to this scale: $0=$ No Pain/Pressure Only; $1=$ Mild Pain; 2 = Moderate Pain; 3 = Severe Pain.

\subsection{Occlusal splints and physiotherapy}

Maxillary stabilization occlusal splints were fabricated with heatpolymerized acrylic resin (Artigos Odontológicos Clássico Ltd., Sao Paulo, Brazil), after diagnostic impressions of the maxillary and mandibular arches were taken with irreversible hydrocolloid (Hydrogum, Zhermack, Badia Polesine, Rovigo, Italy), and then poured in type IV dental stone (Durone, Sao Paulo, Brazil). The intermaxillary relationship was established in habitual maximum intercuspation (HMI) by mounting the mandibular cast in the articulator by tactile and visual intercuspation (Hamata et al., 2009).

Patients were informed to wear the splints at night. All occlusal splints fully covered the maxillary dental arch and had an approximate 2-mm thickness in the molar regions, as well as bilateral and simultaneous occlusal contacts, and immediate disocclusion of the posterior teeth during excursive mandibular movements (Hamata et al., 2009).

All patients received instructions about how to use the heat therapy treatment program at home. (Poindexter, Wright, \& Murchison, 2002). They also received guidelines about sleep and awake bruxism, as well as instructions concerning oral habits such as biting lips, objects, and nails.

\subsection{Bite force measurements}

Bite force was measured with a dynamometer at baseline (initial), after 7 days, and after 30 days of treatment. The IDDK dynamometer (Kratos - Equipamentos Industriais Ltd, Cotia, Sao Paulo, Brazil) was used with $15 \mathrm{~mm}$ in thickness and $1000 \mathrm{~N}$, and adapted to the oral conditions. Training and familiarization with the method was conducted with all patients before the measurements. The evaluation was conducted at the central incisor (anterior) and first molar (posterior) regions on both sides. The patient was asked to bite the device with maximum force 3 times in each region. The records were obtained during $15 \pm 2 \mathrm{~s}$ in a single session with a 2-min interval between the measurements. Three measurements of each area were randomly collected in the morning. The highest value among the three measurements was selected as the maximum bite force (Tortopidis, Lyons, Baxendale, \& Gilmour, 1998). The method error for bite force measurements was determined in 10 patients prior to the experiment. The records were obtained twice a week.

\subsection{Statistical analysis}

Statistical analysis was performed with the SPSS (Statistical Package for the Social Sciences), version 19.0 (IBM SPSS, Chicago, USA). Descriptive statistics (including frequency distributions and percentages) were performed for demographic characteristics of the patients. The Wilcoxon signed-rank non-parametric test was applied to assess the paired difference between the pain levels when the population cannot 
Table 1

Demographic characteristics of the patients $(n=30)$.

\begin{tabular}{|c|c|}
\hline Variables & Female patients treated ${ }^{a}$ \\
\hline Age $(\text { years) })^{\mathrm{b}}$ & $32.9(8.2)$ \\
\hline \multicolumn{2}{|l|}{ Educational level } \\
\hline Incomplete elementary grades & $8(26.7)$ \\
\hline Complete elementary grades & $12(40.0)$ \\
\hline More than complete elementary grades & $10(33.3)$ \\
\hline \multicolumn{2}{|l|}{ Marital Status } \\
\hline Single & $6(20.0)$ \\
\hline Married & $22(73.3)$ \\
\hline Divorced & $2(6.7)$ \\
\hline \multicolumn{2}{|l|}{ Family income } \\
\hline$<3$ times Brazilian minimum wage & $16(53.3)$ \\
\hline$\geq 3$ times Brazilian minimum wage & $14(46.7)$ \\
\hline \multicolumn{2}{|l|}{ Preferably side for chewing } \\
\hline Right & $22(73.3)$ \\
\hline Left & $8(26.7)$ \\
\hline \multicolumn{2}{|l|}{ Contraceptive use } \\
\hline Yes & $18(60.0)$ \\
\hline No & $12(40.0)$ \\
\hline
\end{tabular}

a Values in parentheses are expressed as percentage.

${ }^{\mathrm{b}}$ Mean value (standard deviation).

be assumed to be normally distributed. A two-way repeated-measures analysis of variance (ANOVA) was performed for bite force values to verify any significant difference between regions and periods. Differences revealed by those tests were compared through the Tukey HSD test. Additional analysis related pain level and bite force using scatter plots and linear regression analysis. Statistical significance was set at an alpha level of 0.05 .

\section{Results}

Table 1 shows the distribution of the demographic characteristics of the patients. Table 2 shows the frequency of answers in regard to pain level for each muscle and side, with statistically significant difference using the Wilcoxon signed-rank test $(p<0.05$ at baseline vs. after 30 days). According to Table 2, around $80 \%$ of the patients showed a pain level of 1 or 2 at baseline, and $100 \%$ of the patients showed a pain level of 0 or 1 after 30 days. The results indicated that pain level after treatment was significantly lower than at baseline $(p<0.01)$ for both muscles and sides (Table 2).

ANOVA revealed a significant influence of period and region $(p<0.001)$ on the bite force (Table 3 ). Table 4 shows mean values and standard deviation of bite force. The bite force values were significantly lower in the incisor region when compared with the molar region (both sides), prior to and after treatment $(p<0.001)$. However, there was no significant difference in bite force when comparing right and left sides in the molar area $(p=1.00)$. In the molar region, both sides exhibited significantly higher bite force values at 30 days, when compared to baseline $(p<0.001)$ (Table 4$)$. In addition, there was a significant difference in bite force values on the left side between 7 and 30 days after treatment $(p<0.001)$.

Scatter Plots and linear regression show the relationship between pain levels and bite force at baseline, and change in the period (Figs. 1 and 2). These graphs show that most patients reported pain on only two levels: at a level of 1 and 2 at baseline, and 0 and 1 during the treatment period. Although some graphs and regression suggest that changes in pain level were associated with alterations in bite force only in the temporal muscle (Fig. 1- B), there was no strong correlation or dispersion in the response level points to support that this association would remain true. Therefore, it was not possible to explain the variation in bite force values through the level of pain, despite the significance in some cases. 
Table 3

Results of 2-way repeated-measures ANOVA of bite force.

\begin{tabular}{llllll}
\hline Source & df & SS & MS & F & P \\
\hline Period & 2 & 228449.192 & 114224.596 & 91.168 & $<0.001^{*}$ \\
Erro (Period) & 58 & 72668.446 & 1252.904 & & \\
Region & 2 & 878287.435 & 439143.718 & 66.108 & $<0.001^{*}$ \\
Erro (Region) & 58 & 385280.947 & 6642.775 & & \\
Period"region & 4 & 38703.005 & 9675.751 & 12.194 & $<0.001^{*}$ \\
Erro (Period*Region) & 116 & 92046.506 & 793.504 & & \\
\hline
\end{tabular}

$* \mathrm{P}<0.05$ denotes a statistically significant difference.

Table 4

Mean values and standard deviation of bite force $(\mathrm{N})(\mathrm{n}=30)$.

\begin{tabular}{llll}
\hline \multirow{2}{*}{ Region } & Period & & \\
\cline { 2 - 4 } & Baseline & After 7 days & After 30 days \\
\hline Anterior & $89.3(34.7) \mathrm{Aa}$ & $105.4(29.3) \mathrm{Aa}$ & $119.3(27.8) \mathrm{Aa}$ \\
Right Posterior & $179.9(62.7) \mathrm{Ba}$ & $224.7(88.50) \mathrm{Bab}$ & $272.0(93.6) \mathrm{Bb}$ \\
Left Posterior & $182.2(60.6) \mathrm{Ba}$ & $221.3(73.0) \mathrm{Ba}$ & $273.7(68.7) \mathrm{Bb}$ \\
\hline
\end{tabular}

Different uppercase letters denote statistically significant difference among different regions ( $\mathrm{p}<0.05$ ) and different lowercase letters denote statistically significant difference among different periods $(\mathrm{p}<0.05)$ by Tukey's HSD test.

\section{Discussion}

In this study, a significant increase in the bite force was observed in the molar region after 30 days of treatment, and it remained similar in the incisor region for all periods of analysis. The pain level change was observed over time, however, there was no strong relationship between pain level and bite force.

The present study evaluated the maximum bite force of women with muscular pain and bruxism, prior to and after treatment. The maximum bite force was measured at the central incisor and first molar regions. A significant increase in bite force was observed in the molar region after 30 days of treatment, and it remained similar in the incisor region for all periods of analysis. It is important to consider that several studies reported that men have greater muscular potential than women (Koç et al., 2011; Takaki, Vieira, \& Bommarito, 2014). For this reason, only women were selected to participate in the study, aiming to avoid a heterogeneous and unbalanced sample.

An occlusal splint was indicated to stabilize dental occlusion, protect teeth, and increase the awareness of parafunction, which is possible through the theory of cognitive perception. When occlusal surfaces are covered by the splint, tooth contact on the splint, associated with a change in tongue position, seems to be an important factor that increases the patient's awareness of clenching (Roark, Glaros, \& O'Mahony, 2003).

The significant difference observed between regions and periods (Table 2) is in accordance with previous studies (Regalo et al., 2008;
Tortopidis et al., 1998). Although it was expected that there would be differences between molar and incisor regions, it was hypothesized that the bite force would increase in all regions analyzed after treatment, but this was not verified in the incisor region. As shown in Table 2, bite force was statistically similar prior to and after treatment in the incisor region, even after pain relief, suggesting that the treatment did not increase the bite force, or that the presence of pain did not affect the strength in this region. Bite force in the incisor region was approximately $43 \%$ of the force measured in the molar region after treatment and pain relief, which is in accordance with Ferrario, Sforza, Serrao, Dellavia, and Tartaglia (2004), which found values varying from $40 \%$ to $48 \%$ in healthy young adults. Although this study did not use the same bite force measuring method of previous studies, the bite force values (Table 4) were lower than the mean values reported for healthy patients (from 100.8 to $653.0 \mathrm{~N}$ ) (Cosme, Baldisserotto, Canabarro, \& Shinkai, 2005; Kogawa, Calderon, Lauris, Araujo, \& Conti, 2006) and individuals with bruxism without TMD (from 395.6 to 827 N) (Alkan, Bulut, Arici, \& Sato, 2008; Cosme et al., 2005). Another study (Pereira, Steenks, de Wijer, Speksnijder, \& van der Bilt, 2009) verified a significant increase in bite force after treatment of patients with subacute TMD. However, the values remained lower than those found in asymptomatic patients.

In the present study, the bite force increased over time, which corroborates with another study (Kogawa et al., 2006) that compared the bite force of a control group to myogenic, articular, and mixed TMD groups. In that study, the assessments were only in the regions of the first right and left molars, whereas in the present study the bite force was also measured in the incisor region. Yet, the treatment was effective in increasing bite force in the molar region, corroborating with previous results showing that the molar region concentrates greater force than the anterior region during biting in healthy individuals. It is possible that the significant increase in bite force in the molar region results from the fact that the pain was greater in the masseter (37.29\%) than in the anterior temporal during muscle palpation. It is possible that the presence of pain affected the strength in the molar region more significantly, since masseter muscle power is more evident in this region. With the reduction of muscle pain, there was a significant recovery of bite force in this region.

Although some graphs and regression suggest that changes in pain level were associated with alterations in bite force only in the temporal muscle (Figs. 1-B), there was no strong correlation or dispersion in the response level points to support that conclusion. Therefore, it is not possible to explain the variation in bite force values through the level of pain, despite the significance in some cases. Previous research evaluated patient bite force in the presence of experimental pain, and did not verify a reduction in the maximum bite force of the subjects when compared with the baseline (Kumar, Castrillon, \& Svensson, 2015). The data obtained by Kumar et al. (2015) suggests that not only the presence of pain affects the bite force, but that the ability of the individual to cope with the pain is also important. In addition to the behavioral
(A)

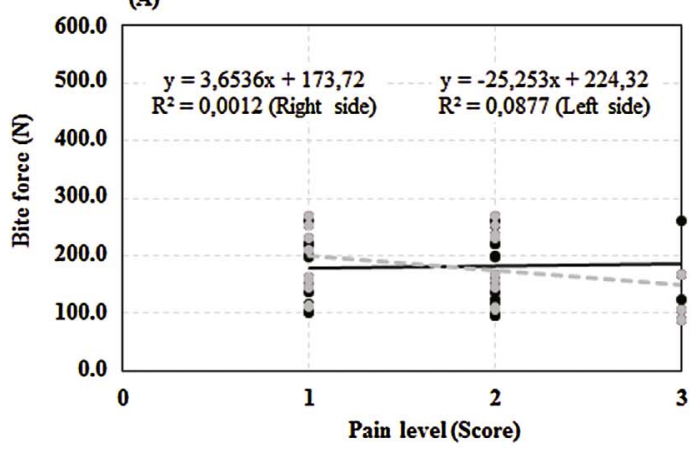

(B)

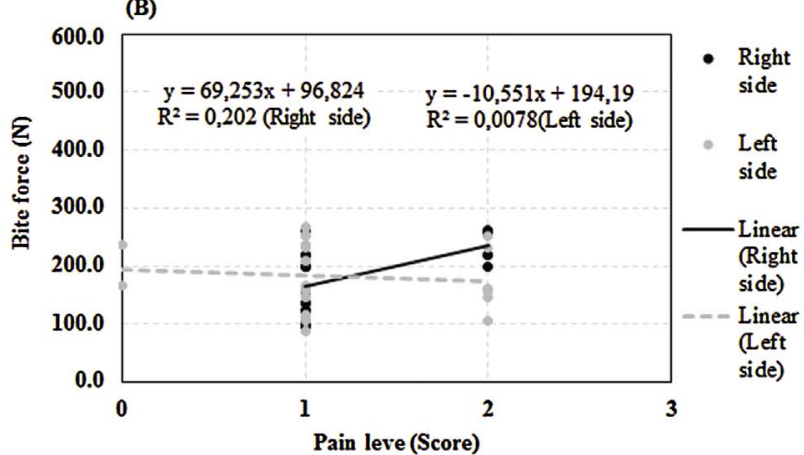

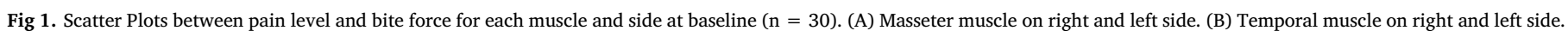


(A)

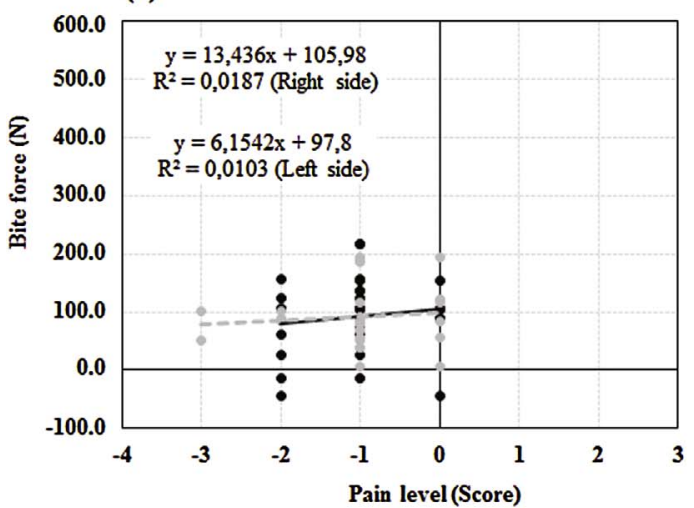

(B)

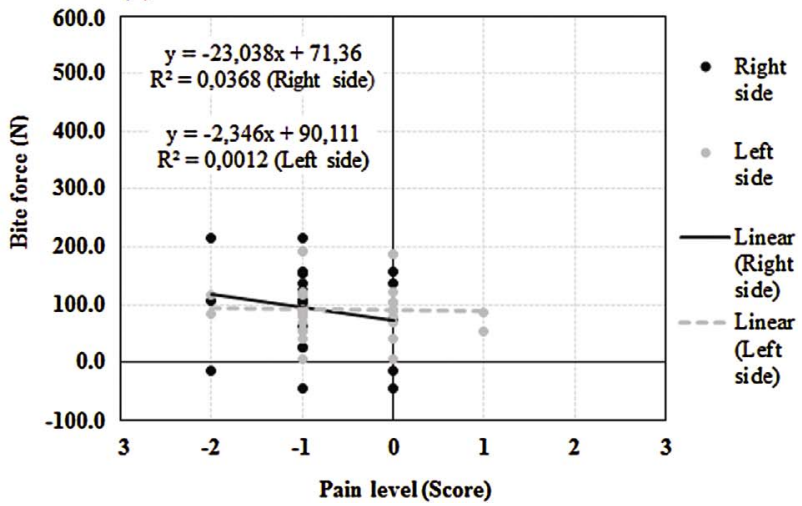

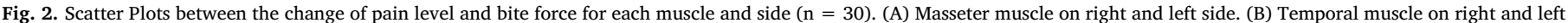
side.

factors against pain, the consistently low levels of pain intensity reported by these patients may have suppressed the level of correlation between pain and bite force as a result of truncation of range. Another important point to be discussed is that the masseter pain could originate from the deep or superficial (head) portion. However, in the present study, researchers evaluated the masseter pain without identifying the affected portion (superficial or deep), which represents a limitation of the study since the pain of the superficial or deep masseter must be investigated separately. Du Brul (1980) divided the masseter muscle in two portions, being the superficial and deep portions, with the deep portion not covered by the superficial portion in an area immediately anterior to the TMJs. This portion can be seen as a triangular muscle field. Researchers verified small differences during mandibular activities due to the difference in the inclination of the muscle fibers in each portion. The deep portion of the masseter muscle pulls the mandible posteriorly with greater force than the superficial masseter (Belser \& Hannam, 1986). A recent study demonstrated, through magnetic resonance, that the deep masseter is likely influenced by changes in anteroposterior bite position (Okada, Yamaguchi, Watanabe, Watanabe, \& Hattori, 2016). This small difference in the function of the masseter portions necessitates the separate evaluation of the superficial and deep portions.

It is important to note that this study has limitations, especially regarding the diagnosis of bruxism. The presence of bruxism identified by patient reports is a problematic method of diagnosis (Raphael et al., 2012). However, it is still used in research due to the high cost of polysomnography. Therefore, it would be necessary to use polysomnography (gold standard) for confirmation of sleep bruxism (Lavigne et al., 2000). For awake bruxism, it would be necessary to use a method for evaluation of muscle contractions during the day (Kawakami, Kumazaki, Manda, Oki, \& Minagi, 2014). Another limitation of this study is the absence of a control group. Without a control, the changes noted here may reflect natural oscillation in the measured variables, regression to the mean, and/or a placebo effect. In addition, the consistently low levels of pain intensity reported by these patients may have suppressed the level of correlation between pain and bite force because of truncation of range. This can be relevant considering that samples with a wider range of pain report some significant association with bite force. Future research should investigate these factors.

Finally, studies are needed to evaluate the relation between pain and bite force, and their interaction with emotional/psychological behavioral aspects, aiming to understand how pain perception can influence the bite force.

\section{Conclusions}

Within the limitations of the present study, it can be concluded that the pain level decreased and the bite force increased in the molar region after treatment. There was no strong correlation or dispersion in the relationship between pain level and bite force in women with myofascial pain and bruxism.

\section{Conflict of interest}

We declare that we have no competing financial interests.

\section{Ethical approval}

The protocol was approved by the Human Research Ethics Committee (Process CEP/286.401).

\section{References}

Abreu, R. A., Pereira, M. D., Furtado, F., Prado, G. P., Mestriner, W., Jr., \& Ferreira, L. M. (2014). Masticatory efficiency and bite force in individuals with normal occlusion. Archives of Oral Biology, 59, 1065-1074.

Ahlberg, K., Savolainen, A., Paju, S., Hublin, C., Partinen, M., Könönen, M., et al. (2008) Bruxism and sleep efficiency measured at home with wireless devices. Journal of Oral Rehabilitation, 35, 567-571.

Alkan, A., Bulut, E., Arici, S., \& Sato, S. (2008). Evaluation of treatments in patients with nocturnal bruxism on bite force and occlusal contact area: A preliminary report. European Journal of Dentistry, 2, 276-282.

Belser, U. C., \& Hannam, A. G. (1986). The contribution of the deep fibers of the masseter muscle to selected tooth-clenching and chewing tasks. Journal of Prosthetic Dentistry, $56,629-635$.

Cosme, D. C., Baldisserotto, S. M., Canabarro, A. S., \& Shinkai, R. S. (2005). Bruxism and voluntary maximal bite force in young dentate adults. The International Journal of Prosthodontics, 18, 328-332.

de Leeuw, R., \& Klasser, G. D. (2013). Orofacial pain: Guidelines for assessment, diagnosis and management (5th ed.). Hanover Park: Quintessence Pub Co (Chapter 8).

Du Brul, E. L. (1980). Sicher's oral anatomy (7th ed.). St. Louis: The C:V Mosby Co.

Dworkin, S. F., \& LeResche, L. (1992). Research diagnostic criteria for temporomandibular disorders: Review, criteria, examinations and specifications, critique. Journal of Craniomandibular Disorders: Facial \& Oral Pain, 6, 301-355.

English, J. D., Buschang, P. H., \& Throckmorton, G. S. (2002). Does malocclusion affect masticatory performance? The Angle Orthodontist, 72, 21-27.

Faul, F., Erdfelder, E., Lang, A. G., \& Buchner, A. G. (2007). G*Power 3: A flexible statistical power analysis program for the social, behavioral, and biomedical sciences. Behavior Research Methods, 39, 175-191.

Fernandes, C. P., Glantz, P. O., Svensson, S. A., \& Bergmark, A. (2003). A novel sensor for bite force determinations. Dental Materials, 19, 118-126.

Ferrario, V. F., Sforza, C., Serrao, G., Dellavia, C., \& Tartaglia, G. M. (2004). Single tooth bite forces in healthy young adults. Journal of Oral Rehabilitation, 31, 18-22.

Gomes, C. A., El Hage, Y., Amaral, A. P., Politti, F., \& Biasotto-Gonzalez, D. A. (2014). Effects of massage therapy and occlusal splint therapy on electromyographic activity and the intensity of signs and symptoms in individuals with temporomandibular disorder and sleep bruxism: A randomized clinical trial. Chiropractic \& Manual Therapies, 22, 43 .

Hamata, M. M., Zuim, P. R., \& Garcia, A. R. (2009). Comparative evaluation of the efficacy of occlusal splints fabricated in centric relation or maximum intercuspation in temporomandibular disorders patients. Journal of Applied Oral Science, 17, 32-38.

Kawakami, S., Kumazaki, Y., Manda, Y., Oki, K., \& Minagi, S. (2014). Specific diurnal EMG activity pattern observed in occlusal collapse patients: Relationship between diurnal bruxism and tooth loss progression. Public Library Of Science, 9, e101882.

Klasser, G. D., Greene, C. S., \& Lavigne, G. J. (2010). Oral appliances and the management of sleep bruxism in adults: A century of clinical applications and search for 
mechanisms. The International Journal of Prosthodontics, 23, 453-462.

Koç, D., Doğan, A., \& Bek, B. (2011). Effect of gender, facial dimensions: Body mass index and type of functional occlusion on bite force. Journal of Applied Oral Science, 19, 274-279.

Kogawa, E. M., Calderon, P. S., Lauris, J. R., Araujo, C. R., \& Conti, P. C. (2006). Evaluation of maximal bite force in temporomandibular disorders patients. Journal of Oral Rehabilitation, 33, 559-565.

Kumar, A., Castrillon, E., \& Svensson, P. (2015). Can experimentally evoked pain in the jaw muscles or temporomandibular joint affect anterior bite force in humans? Journal of Oral \& Facial Pain and Headache, 29, 31-40.

Lavigne, G., Zucconi, M., Castronovo, C., Manzini, C., Marchettini, P., \& Smirne, S. (2000). Sleep arousal response to experimental thermal stimulation during sleep in human subjects free of pain and sleep problems. Pain, 84, 283-290.

Lobbezoo, F., Ahlberg, J., Glaros, A. G., Kato, T., Koyano, K., Lavigne, G. J., et al. (2013). Bruxism defined and graded: An international consensus. Journal of Oral Rehabilitation, 40, 2-4.

Mancuso, D. N., Goiato, M. C., Gennari Filho, H., \& Gomes, E. A. (2008). Bite force and masticatory efficiency in implant-retained dentures: Literature review. Dentistry Today, 27, 56-58.

Okada, T., Ikebe, K., Inomata, C., Takeshita, H., Uota, M., Mihara, Y., et al. (2014). Association of periodontal status with occlusal force and food acceptability in 70year-old adults: From sonic study. Journal of Oral Rehabilitation, 41, 912-919.

Okada, C., Yamaguchi, S., Watanabe, Y., Watanabe, M., \& Hattori, Y. (2016). Evaluation of masticatory activity during unilateral single tooth clenching using muscle functional magnetic resonance imaging. Journal of Oral Rehabilitation, 43, 583-590.

Paesani, D. A., Lobbezoo, F., Gelos, C., Guarda-Nardini, L., Ahlberg, J., \& Manfredini, D. (2013). Correlation between self-reported and clinically based diagnoses of bruxism in temporomandibular disorders patients. Journal of Oral Rehabilitation, 40, 803-809.

Palinkas, M., Nassar, M. S., Cecílio, F. A., Siéssere, S., Semprini, M., Machado-de-Sousa, J. P., et al. (2010). Age and gender influence on maximal bite force and masticatory muscles thickness. Archives of Oral Biology, 55, 797-802.

Pereira, L. J., Steenks, M. H., de Wijer, A., Speksnijder, C. M., \& van der Bilt, A. (2009). Masticatory function in subacute TMD patients before and after treatment. Journal of
Oral Rehabilitation, 36, 391-402.

Pereira-Cenci, T., Pereira, L. J., Cenci, M. S., Bonachela, W. C., \& Del Bel Cury, A. A. (2007). Maximal bite force and its association with temporomandibular disorders. Brazilian Dental Journal, 18, 65-68.

Pintado, M. R., Anderson, G. C., DeLong, R., \& Douglas, W. H. (1997). Variation in tooth wear in young adults over a two-year period. Journal of Prosthetic Dentistry, 77, 313-320.

Poindexter, R. H., Wright, E. F., \& Murchison, D. F. (2002). Comparison of moist and dry heat penetration through orofacial tissues. Cranio, 20, 28-33.

Raphael, K. G., Sirois, D. A., Janal, M. N., Wigren, P. E., Dubrovsky, B., Nemelivsky, L. V., et al. (2012). Sleep bruxism and myofascial temporomandibular disorders: A laboratory-based polysomnographic investigation. The Journal of the American Dental Association, 143, 1223-1231.

Regalo, S. C., Santos, C. M., Vitti, M., Regalo, C. A., de Vasconcelos, P. B., Mestriner, W., Jr., et al. (2008). Evaluation of molar and incisor bite force in indigenous compared with white population in Brazil. Archives of Oral Biology, 53, 282-286.

Roark, A. L., Glaros, A. G., \& O'Mahony, A. M. (2003). Effects of interocclusal appliances on emg activity during parafunctional tooth contact. Journal of Oral Rehabilitation, 30, 573-577.

Shimada, A., Baad-Hansen, L., \& Svensson, P. (2015). Effect of experimental jaw muscle pain on dynamic bite force during mastication. Archives of Oral Biology, 60, 256-266.

Svensson, P., Burgaard, A., \& Schlosser, S. (2001). Fatigue and pain in human jaw muscles during a sustained, low-intensity clenching task. Archives of Oral Biology, 46, 773-777.

Takaki, P., Vieira, M., \& Bommarito, S. (2014). Maximum bite force analysis in different age groups. International Archives of Otorhinolaryngology, 18, 272-276.

Tortopidis, D., Lyons, M. F., Baxendale, R. H., \& Gilmour, W. H. (1998). The variability of bite force measurement between sessions: In different positions within the dental arch. Journal of Oral Rehabilitation, 25, 681-686.

Wahlund, K., Nilsson, I. M., \& Larsson, B. (2015). Treating temporomandibular disorders in adolescents: A randomized, controlled: Sequential comparison of relaxation training and occlusal appliance therapy. Journal of Oral \& Facial Pain and Headache, $29,41-50$. 